EPJ Web of Conferences 40, 08008 (2013)

DOI: $10.1051 /$ epjconf/20134008008

(C) Owned by the authors, published by EDP Sciences, 2013

\title{
Irreversible magnetic properties of carbon nanoparticles
}

\author{
E. Lähderanta ${ }^{1}$, A.V. Lashkul ${ }^{1}$, K.G. Lisunov ${ }^{1,2}$, D.A. Zherebtsov ${ }^{3}$, D.M. Galimov ${ }^{3}$, and A.N. Titkov ${ }^{4}$ \\ ${ }^{1}$ Department of Mathematics and Physics, Lappeenranta University of Technology, PO Box 20, FIN-53851 \\ Lappeenranta, Finland \\ ${ }^{2}$ Institute of Applied Physics, Academy of Sciences of Moldova, Academiei Str. 5, MD-2028 Kishinev, \\ Moldova \\ ${ }^{3}$ South Ural State University, 454080 Chelyabinsk, Russia \\ ${ }^{4}$ A.F. Ioffe Physico-Technical Institute, 194021 St. Petersburg, Russia
}

\begin{abstract}
Magnetic properties of powder and glassy samples with carbon nanoparticles, not intentionally doped and doped with $\mathrm{Ag}$, $\mathrm{Au}$ and $\mathrm{Co}$, are investigated at temperatures $T \sim 3-300 \mathrm{~K}$ in magnetic fields $B$ up to $5 \mathrm{~T}$. Magnetization $M(T)$ exhibits in low fields of $B=1-50 \mathrm{mT}$ a strong irreversibility, which is suppressed above $B \sim 1 \mathrm{~T}$. The high-temperature $(T \sim 200-300 \mathrm{~K})$ dependence of $M$ on $B$ demonstrates a saturation above $B \sim 2 \mathrm{~T}$. Magnetic hysteresis is observed already at $300 \mathrm{~K}$, exhibiting a power-law temperature decay of the coercive field. Analysis of the experimental data suggests a concentration of the magnetization close to the surface of the carbon nanoparticles. This is consistent with the origin of magnetism in nanocarbon due to intrinsic surface defects. Deviations of macroscopic and microscopic parameters in the Co-doped sample from those in the other samples imply an influence of the Co ions and/or clusters.
\end{abstract}

\section{Introduction}

Magnetic properties of carbon-based materials attract considerable attention both due to scientific and practical reasons [1]. Indeed, the fact that such materials, being metal-free or containing only negligible amounts of metallic impurities, demonstrate a ferromagnetic (FM) behavior even above the room temperature, is intriguing by itself. On the other hand, a broad utilization area, stretching from spintronics and light metals in techniques to applications for biological and medical purposes, is expectable for such unusual carbon-based magnetism [1].

The most known bulk carbon modifications as graphite and diamond, containing negligible intrinsic disorder, exhibit only diamagnetism [2]. Therefore, the unconventional magnetic properties of carbon-based materials are attributable to defects or disorder introduced into an ordered host matrix. In particular, this has been demonstrated directly by intentional incorporation of defects by proton irradiation of graphite, leading to pronounced FM properties [1, 3].

Experimental evidence of the intrinsic magnetism in defect-rich carbon materials [1-3] is accompanied by theoretical predictions of the FM behavior in such structures as negatively-curved graphite surface [4], a mixture of carbon atoms with alternation of $s p^{2}-s p^{3}$ bonds [5], the graphene zigzag edges [6] and a disordered graphite with random atomic defects [7]. Predicted values of the localized magnetic moments, $\mu_{\mathrm{L}} \sim 1-2 \mu_{\mathrm{B}}$, associated with intrinsic defects or disorder $[8,9]$, are similar to those obtained experimentally in highlyoriented pyrolitic graphite, $\mu_{\mathrm{L}} \sim 0.2-1.5 \mu_{\mathrm{B}}$ per defect at an inter-defect distance of $\sim 0.5-4 \mathrm{~nm}[10]$.

Here we investigate magnetic properties of a system of carbon nanoparticles, which represents an interesting case of the defect-rich carbon media due to numerous surface defects and high surface-to volume ratio.

\section{Results and discussion}

\subsection{Experimental results}

Not intentionally doped powder carbon sample (\# 1) and porous glassy carbon samples doped with 0.00123 mass $\%$ of $\mathrm{Ag}(\# 2), 0.004$ mass \% of $\mathrm{Au}(\# 3)$ and 0.117 mass $\%$ of Co (\# 4) are used for the investigations below. The preparation of the glassy samples is described in [11], yielding no evidence for clustering of the metallic impurity excluding probably in \# 4. The atomic-force microscopy data (not shown) give evidence for presence of a system of carbon nanoparticles in all the investigated samples with a broad size distribution, characterized by the average, $R_{\mathrm{av}} \sim 60 \mathrm{~nm}$, and the maximum, $R_{\max } \sim 110$ $\mathrm{nm}$, particle radii.

This is an Open Access article distributed under the terms of the Creative Commons Attribution License 2.0, which permits unrestricted use, distribution, and reproduction in any medium, provided the original work is properly cited. 

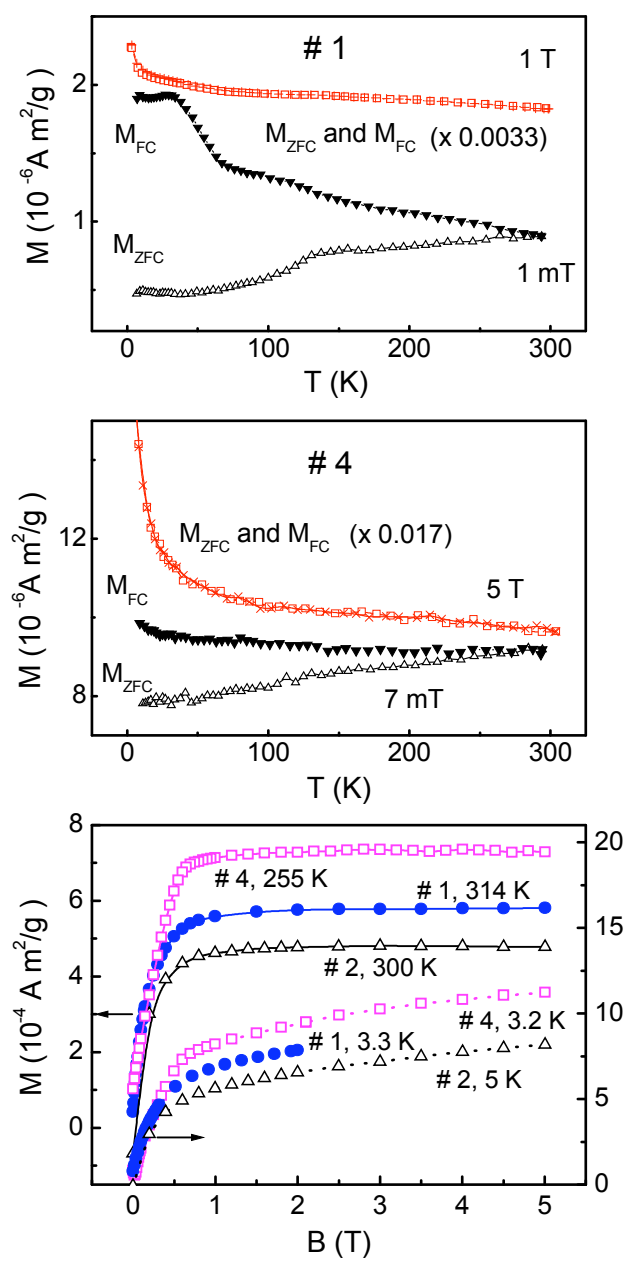

Fig. 1. The plots of $M_{\mathrm{ZFC}}$ and $M_{\mathrm{FC}}$ vs. $T$ (top and middle panels) and the dependence of $M$ on $B$ (bottom panel).

Temperature dependence of the magnetization, $M$, per unit sample mass in the fields of $B=1-50 \mathrm{mT}$ (the examples for \# 1 and \# 4 are shown in the top and middle panels of figure 1, respectively) exhibits a strong magnetic irreversibility. This means deviation of the magnetization, measured after cooling a sample in zero magnetic field, $M_{\mathrm{ZFC}}(T)$, from that obtained after cooling in a field, $M_{\mathrm{FC}}(T)$. However, the magnetic irreversibility decreases with increasing the field and disappears above $B \sim 1 \mathrm{~T}$. The dependence of $M$ on $B$ exhibits a saturation at $T \sim 200-300 \mathrm{~K}$, which does not take place at $T \sim 3-$ $5 \mathrm{~K}$ (bottom panel of figure 1). Magnetic hysteresis is observed already at the room temperature. The hysteresis curves in \# 2 and \# 3 resembles the image, observed in \# 1 (top panel of figure 2), but differ considerably from that of \# 4 in the middle panel of figure 2 .

\subsection{Macroscopic magnetic behavior}

The magnetic irreversibility in the top and middle panels of figure 1 is typical of an assembly of small magnetic particles with a broad size distribution, provided that the inequalities $B<B_{\mathrm{K}}$ and $T<T_{\mathrm{b}}$ are fulfilled. Here $B_{\mathrm{K}}$ is the


Fig. 2. The hysteresis curves (top and middle panels) and the plots of $B_{\mathrm{c}}$ vs. $T^{n}$ (bottom panel).

mean anisotropy field and $T_{\mathrm{b}} \approx K V /\left(25 k_{\mathrm{B}}\right)$ is the blocking temperature, where $K$ is the volume density of the anisotropy energy and $V$ is the particle volume [12]. The inequalities above characterize the blocked or stable regime, in which thermal excitations cause only a slow rotation of the particle moments leading to a glassy magnetic state. Application of the magnetic field leads to diminution of the anisotropy barriers and to damping of the magnetic irreversibility with increasing $B$, which vanishes at $B>B_{\mathrm{K}}[12]$. Therefore, the anisotropy field in our case can be estimated as $B_{\mathrm{K}} \approx 1 \mathrm{~T}$ in \# $1-3$ and lying between $B_{\mathrm{K}} \sim 1-5 \mathrm{~T}$ in \# 4 . Because the onset of divergence of $M_{\mathrm{ZFC}}(T)$ and $M_{\mathrm{FC}}(T)$ takes place already at $300 \mathrm{~K}, T_{\mathrm{b}}$ lies definitely above the room temperature. This is consistent with the rapid saturation of $M(B)$ at $T$ $250-300 \mathrm{~K}$ in the bottom panel of Fig. 1, characteristic of blocking of the relatively large magnetic particles with $R \sim R_{\mathrm{av}}$. On the other hand, an evident deviation from saturation at low $T \sim 3-5 \mathrm{~K}$ (bottom panel of Fig. 1), as well as the low-temperature upturn of both $M_{\mathrm{ZFC}}(T)$ and 
$M_{\mathrm{FC}}(T)$ at $B \sim 1-5 \mathrm{~T}$ in the top and middle panels of Fig. 1, are noticeable. These features give evidence for presence of the magnetic particles with $R<<R_{\mathrm{av}}$, which are small enough to be unblocked down to the lowest temperatures providing a paramagnetic or superparamagnetic contribution to the net magnetization. Therefore, such particles do not contribute to such macroscopic parameters as the saturation magnetization, $M_{\mathrm{s}}(0)$, and the coercive field, $B_{\mathrm{c}}$, characterizing the blocked nanoparticle system. Their effect can be excluded by fitting the plots of $M(T)$ at $B \geq 2 \mathrm{~T}$ with the Curie-Weiss-like law, $M(T) \approx M_{\mathrm{s}}(0)+C B /(T-\theta)$, giving the values of $M_{\mathrm{s}}(0) \approx 5.6,4.9,4.7$ and 7.2 (in units of $10^{-4} \mathrm{Am}^{2} / \mathrm{g}$ ) for \# 1, 2, 3 and 4, respectively.

The hysteresis loop in the top panel of figure 2 is typical of an assembly of magnetic nanoparticles in the blocked regime. On the other hand, the behavior in the middle panel of figure 2 suggests a FM contribution of the Co ions and/or clusters. In both cases a substantial decay of $B_{\mathrm{c}}$ with $T$ is observed, which is analysed with a general expression, $B_{\mathrm{c}}(T)=B_{\mathrm{c}}(0)\left[1-\left(T / T_{\mathrm{b}}\right)^{n}\right]$. Here $B_{\mathrm{c}}$ (0) is the low-temperature coercive field and $n=1 / 2$ and $2 / 3$ [12] for the magnetization reversal, realized by coherent rotation and curling, respectively, in a system of the single-domain (SD) particles. The value of $n=1$ is connected to domain wall effects, when the conditions of the SD regime are violated e. g. due to increase of the particle size [13]. The plots of $B_{\mathrm{c}}$ vs. $T^{n}$, shown in the bottom panel of Fig. 2, exhibit the best linearization with the values of $n \approx 0.80-0.81$, yielding $T_{\mathrm{b}} \approx 570,405$ and $580 \mathrm{~K}$ and $B_{\mathrm{c}}(0) \approx 36,52$ and $80 \mathrm{mT}$ for \# 1 , \# 3 and \# 4, respectively. The values of $T_{\mathrm{b}}$ lying well above the room temperature are consistent with the magnetic field and temperature dependences of the magnetization, discussed above. On the other hand, the relation $2 / 3<n<1$ indicates a crossover of the SD curling mode of the magnetization reversal and a multidomain regime. The curling mode is supported by the relation $B_{\mathrm{c}}(0)<<B_{\mathrm{K}}$ (for coherent rotation these parameters should be comparable), whereas the crossover is substantiated by relatively large values of $R_{\mathrm{av}}$ and $R_{\max }$, accompanied with the broad size distribution of the carbon nanoparticles, as mentioned in the beginning of Section 2.1.

\subsection{Microscopic analysis}

The microscopic parameters characterizing the magnetic properties of carbon nanoparticles and the internal localized magnetic moments can be obtained with the analysis of such macroscopic data as $M_{\mathrm{s}}(0), B_{\mathrm{c}}(0)$ and $T_{\mathrm{b}}$ found in Section 2.2 above. The analysis have been performed using a conventional phenomenological treatment of a system of small and almost spherical magnetic particles, suggesting their additive contributions to the net magnetic response and taking into account an expected concentration of the localized magnetic moments within a thin near-surface particle volume with thickness $h<<R$. Expressions for a critical radius $r_{\text {sd }}$ of the SD particle and a critical radius $r_{\mathrm{c}}$ of curling [13] have been used, as well, taking into account the criteria
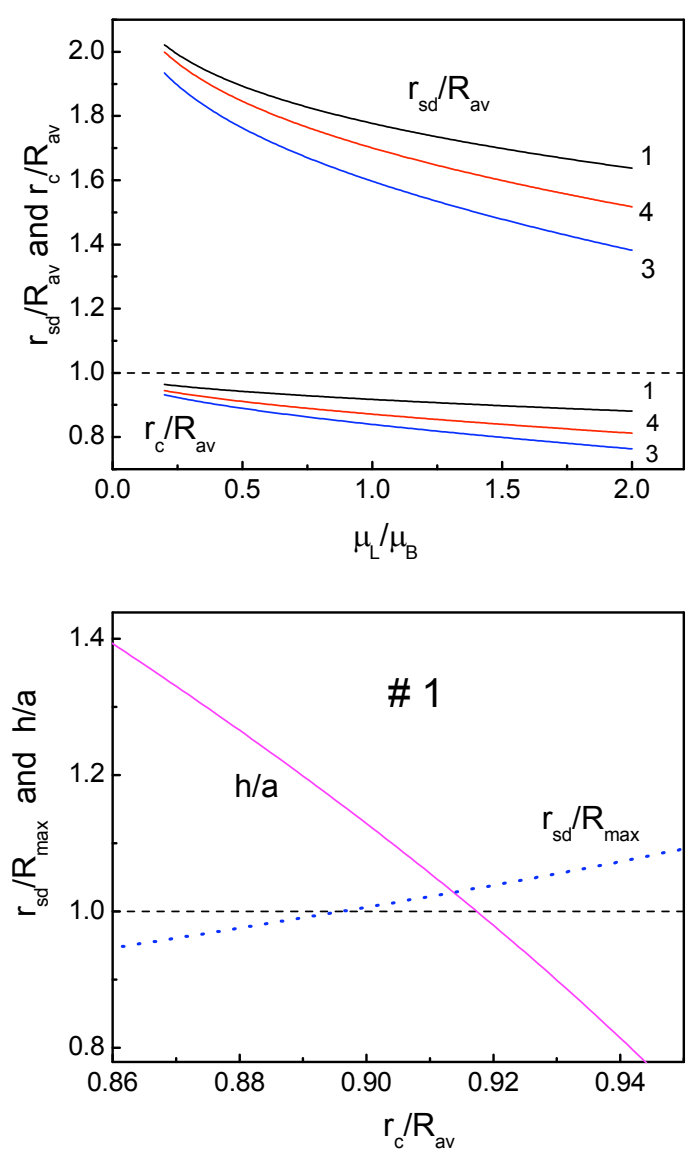

Fig. 3. The dependences of $r_{\mathrm{sd}} / R_{\mathrm{av}}$ and $r_{\mathrm{c}} / R_{\mathrm{av}}$ on $\mu_{\mathrm{L}} / \mu_{\mathrm{B}}$ for \# 1, \# 3 and \# 4 (top panel). The plots of $r_{\mathrm{sd}} / R_{\max }$ vs. $r_{\mathrm{c}} / R_{\mathrm{av}}$ and $h / a$ vs. $r_{\mathrm{c}} / R_{\mathrm{av}}$ (bottom panel).

$R<<r_{\mathrm{sd}}$ and $R>>r_{\mathrm{c}}$ for the $\mathrm{SD}$ regime and the curling magnetization reversal, respectively [13].

Calculations of $r_{\mathrm{sd}} / R_{\mathrm{av}}$ and $r_{\mathrm{c}} / R_{\mathrm{av}}$ at $\mu_{\mathrm{L}}=0.2-2 \mu_{\mathrm{B}}$ (taking into account the experimental and theoretical values of $\mu_{\mathrm{L}}$ cited in the Introduction), made by putting $h$ $=a$, where $a$ is the average distance between the internal localized magnetic moments of the particle, are presented in the top panel of figure 3. It is evident that the relations $R_{\mathrm{av}} \leq r_{\mathrm{sd}}$ and $R_{\mathrm{av}} \geq r_{\mathrm{c}}$ are satisfied reasonably. This agrees well with the results of analysis of the coercivity field (see the end of Section 2.2). The values of $a\left(\mu_{\mathrm{L}}\right)$, found within $\sim 0.3-1.2 \mathrm{~nm}$, are consistent with strong FM interactions between the localized moments [5-9].

Calculations in the bottom panel of figure 3 are performed by putting $\mu_{\mathrm{L}}=\mu_{\mathrm{B}}$ and by variation of $r_{\mathrm{c}}$, where the plots of $r_{\mathrm{sd}} / R_{\max }$ vs. $r_{\mathrm{c}} / R_{\mathrm{av}}$ and $h / a$ vs. $r_{\mathrm{c}} / R_{\mathrm{av}}$ are compared with the horizontal line of unity. Due to the relations $r_{\mathrm{sd}} / R_{\max } \geq 1$ and $h / a \geq 1$, the interval of acceptable values of the ratios $r_{\mathrm{sd}} / R_{\max }$ and $h / a$ is presented by the plots of $r_{\mathrm{sd}} / R_{\max }$ vs. $r_{\mathrm{c}} / R_{\mathrm{av}}$ and $h / a$ vs. $r_{\mathrm{c}} / R_{\mathrm{av}}$ lying above unity. At the same time, the corresponding interval of $r_{\mathrm{c}} / R_{\mathrm{av}}$ lies between the values of $X_{\min } \equiv\left(r_{\mathrm{c}} / R_{\mathrm{av}}\right)_{\min }$ and $X_{\max } \equiv\left(r_{\mathrm{c}} / R_{\mathrm{av}}\right)_{\max }$, which are determined by intersections of these plots with the constant line of unity, respectively. Hence, the values of $a$ 
and $h$ can be found within the error, given by the difference of $X_{\min }$ and $X_{\max }$, where $X_{\min } \leq X_{\max }$. As follows from the bottom panel of figure 3, for \# 1 the condition of $X_{\min } \leq X_{\max }$ is fulfilled with the difference of $\sim 2 \%$ between $X_{\min }$ and $X_{\max }$. Although in the other samples (not shown) we have $X_{\min }>X_{\max }$, the difference between them $\sim 1-5 \%$ is insignificant. Finally, we obtain the relation $h \approx a$ with the error of $\sim 15 \%$ and $20 \%$ in $\# 1$ and \# 3, respectively, which is reduced down to $\sim 4 \%$ and $3 \%$ in \# 2 and \# 4 , respectively. The values of $a \approx h$ collected in Table 1 are in agreement with the relation of $h / R_{\mathrm{av}}<<1$ supposed above. In addition, the maximim particle moment, $\mu_{\max }$, the saturation magnetization, $M_{\mathrm{s}}{ }^{*}$, and the exchange stiffness constant, $A$, of the particle material are obtained in this way (Table 1). It can be seen that all the microscopic parameters above, as well as the macroscopic data of $M_{\mathrm{s}}(0)$ and $B_{\mathrm{c}}(0)$ in Section 2.2, in \# 4 deviate from the corresponding data in the other samples, suggesting influence of the Co ion moments and/or clusters.

Table 1. Microscopic parameters $a, \mu_{\max }, M_{\mathrm{s}}^{*}$ and $A$.

\begin{tabular}{|c|c|c|c|c|}
\hline Sample & $\begin{array}{c}a, \\
\mathrm{~nm}\end{array}$ & $\begin{array}{c}\mu_{\max }, \\
10^{5} \mu_{\mathrm{B}}\end{array}$ & $\begin{array}{c}M_{\mathrm{s}}^{*}, \\
\mathrm{emu} / \mathrm{cm}^{3}\end{array}$ & $\begin{array}{c}A, \\
10^{-6} \mathrm{erg} / \mathrm{cm}\end{array}$ \\
\hline$\# 1$ & 0.74 & 3.5 & 44 & 5.0 \\
\hline$\# 2$ & 0.79 & 3.1 & 36 & 3.1 \\
\hline$\# 3$ & 0.81 & 3.0 & 33 & 2.5 \\
\hline$\# 4$ & 0.65 & 4.5 & 67 & 9.6 \\
\hline
\end{tabular}

Finally, the FM Curie temperature, $T_{\mathrm{C}}$, can be estimated with the equation

$T_{\mathrm{C}}=4 \pi J S^{2}\left\{\ln \left[T_{\mathrm{C}} /\left(J S \Delta_{0}\right)\right]+4 \ln \left(4 \pi J S^{2} / T_{\mathrm{C}}\right)+C_{\mathrm{F}}\right\}$,

where $S=1 / 2, \Delta_{0}=10^{-4}$ is the dimensionless spin-wave gap, $C_{\mathrm{F}} \approx 0$ and $J \approx A a /\left(2 S^{2}\right)$ is the FM exchange constant [14]. As can be seen in figure 4 the values of $J$ exhibit an expectable substantial decrease with $a$, whereas $T_{\mathrm{C}}$ exceeds $T_{\mathrm{b}}$ at any $a$, although the case of Codoping may not match this model explicitly due to contribution of the Co ion moments, as mentioned above. Anytime, the relation of $T_{\mathrm{C}}>T_{\mathrm{b}}$ is typical of FM materials and demonstrates the reliability of the values of $T_{\mathrm{b}}$ obtained in the investigated nanocarbon samples.

\section{Conclusions}

Magnetic properties of carbon nanoparticles have been investigated. Magnetic irreversibility, saturation of the magnetization and magnetic hysteresis typical of an assembly of small magnetic particles in the blocked regime have been observed. In addition, the microscopic analysis demonstrates concentration of the magnetization close to the surface of the particles. This is consistent with the origin of magnetism in nanocarbon due to intrinsic near-surface defects. However, in the Co-doped

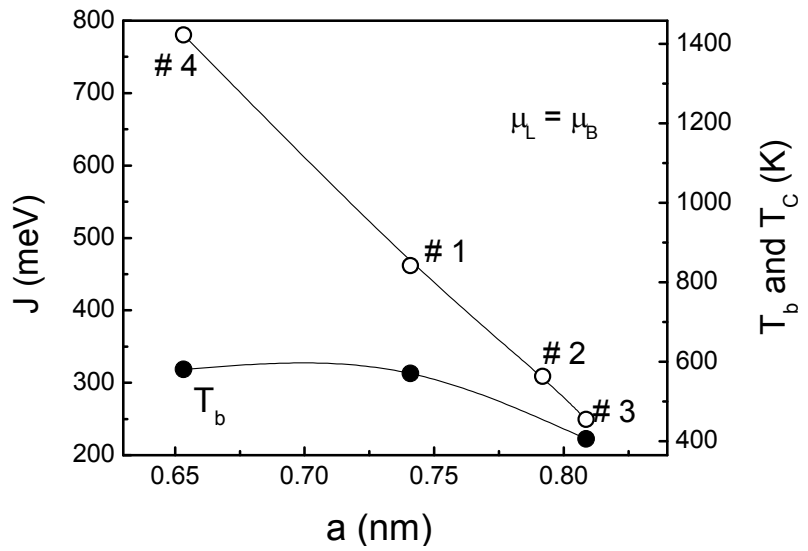

Fig. 4. The plots of $T_{\mathrm{b}}$ vs. $a(\bullet$, right axis), $J$ vs. $a$ (o, left axis) and $T_{\mathrm{C}}$ vs. $a$ (o, right axis).

sample the obtained macroscopic and microscopic magnetic parameters deviate from those in other samples, suggesting influence of Co ions and/or clusters.

\section{References}

1. T. Makarova, F. Palacio (editors), Carbon-Based Magnetism (Elsevier, North-Holland, 2006)

2. P. Stamenov, J.M.D. Coey, J. Magn. Magn. Mater. 290-291, 279 (2005)

3. Barzola-Quiquia J, Höhne R, Rothermel M, Setzer A, Esquinazi P and Heera V 2008 Eur. Phys. J. B 61 127

4. Park N, Yoon M, Berber S, Ihm J, Osawa E and Tomanek D 2003 Phys. Rev. Lett. 91237204

5. Ovchinnikov A A and Spektor V N 1988 Synth. Mather. 27 B615

6. Yazyev O V and Katsnelson M I 2008 Phys. Rev. Lett.100 047209

7. Yazyev O V 2008 Phys. Rev. Lett. 10137203

8. Faccio R, Pardo H, Denis P A, Oeiras R Y, AraujoMoreira F M, Verissimo-Alves M and Mombru A W 2008 Phys. Rev. B 77035416

9. Yazyev O V and Helm L 2007 Phys. Rev. B 75 125408

10. Červenka J, Katsnelson M I and Flipse C F J 2009 Nature Physics 5840

11. E. Lähderanta, A.V. Lashkul, K.G. Lisunov, D.A. Zherebtsov, D.M. Galimov, A.N. Titkov, IOP Conf. Ser.: Mater. Sci. Eng. (to be published)

12. Sun L, Hao Y, Chien C L and Searson P 2005 IBM J. Res. \& Dev. 4979

13. Frei E H, Shtrikman S and Trevis D 1957 Phys. Rev. 106446

14. Irkhin V Y, Katanin A A and Katsnelson M I 1999 Phys. Rev. B 601082 